\title{
A New Empirical Model for Indoor Propagation Prediction ${ }^{\dagger}$
}

\author{
K.W. Cheung ${ }^{+}$, J.H.M. Sau ${ }^{++}$and, R.D. Murch ${ }^{\frac{1}{+}}$, Member IEEE, \\ Department of Electrical and Electronic Engineering, \\ Hong Kong University of Science and Technology, \\ Clear Water Bay, Kowloon, \\ HONG KONG. \\ September 1997
}

\begin{abstract}
In this paper a new empirical model for indoor propagation prediction is presented. The inspiration for our model is to enhance existing empirical models for indoor propagation prediction by incorporating additional phenomena suggested by electromagnetic techniques such as the Uniform Theory of Diffraction but still retain the straightforwardness of the empirical approach. The advantage is that computation time for indoor propagation prediction is low without greatly compromising prediction accuracy. Comparisons of our predicted results to measurements indicate that improvements in accuracy over conventional empirical models are achieved.
\end{abstract}

$\dagger$ Supported by the Hong Kong Research Grants Council and Hutchison Telecom.

Corresponding Author. Email: eermurch@ee.ust.hk. Tel: (+852) 2358 7044. Fax: (+852) 23581485.

+ Now with Hong Kong Telecom, Hong Kong

++ Now with Motorola Information Products and Services Group, Canada 


\section{Introduction}

There has been considerable interest recently in propagation prediction for indoor environments. The reason is that indoor propagation prediction is becoming very useful to mobile telephone operators that provide services in large cities where many subscribers are pedestrians demanding that coverage be provided within buildings, shopping malls and train stations. [1, 2]. Combine this with the extremely high density of subscribers within these cities (making the radio spectrum resource very scare) and it can be deduced that mobile operator profit margins depend highly on maximizing capacity and hence performing accurate indoor propagation prediction.

Previously two approaches to indoor propagation prediction have been investigated. In one approach propagation models related to those for describing free space propagation are empirically or statistically fitted to measurement data $[3,4]$. The resulting models are generally straightforward to apply and prediction results can be computed quickly. A limitation however is that measurement data must first be obtained in order for the empirical parameters to be determined. A further limitation is that the propagation models can generally only model propagation phenomena which does not depart radically from propagation in free space.

In the alternative approach, electromagnetic theory is applied more rigorously using ray tracing techniques such as the Uniform Theory of Diffraction (UTD) and invokes detailed site specific information about the particular building $[5,6,7,8]$. In principle propagation predictions can be performed without first performing propagation measurements. Furthermore the predictions based on ray tracing can predict intricate propagation phenomena such as diffraction and reflection. However computation times on personal computers can be large and hence the predictions cannot be performed interactively or with algorithms for optimizing basestation locations [9]. Another area for concern is that although the ray tracing techniques are based on formal electromagnetic theory, tweaking of the permittivity constants is employed in order to get a good match with the measurement results. Thus in effect these models have been reduced to an empirical model thereby rendering the advantages over empirical models questionable.

In this paper we propose a new empirical propagation model which incorporates much of the propagation phenomena that is suggested by electromagnetic theory such as UTD but still retains the straightforwardness of the empirical approach. A potential advantage of this is that the empirical factors required 
in the model can be related closely to theoretical derivations so that fitting or optimization of the model to propagation data will not necessarily be required. Another advantage of this is that computation time can be kept to a minimum so that predictions can be performed interactively on a computer without a significant reduction in prediction accuracy [9]. In a sense our model can be thought of as being in the middle ground between empirical and ray tracing techniques.

In section 2 we provide the necessary background for our developments and in section 3 the details of the new empirical model are given. Propagation predictions from the new model are compared to actual measurements in section 4. In addition discussions on the significance of the results and their performance compared to an existing empirical model is provided. Conclusions are included in section 5.

\section{Background}

We define path loss in $\mathrm{dB}$ as

$$
P L(d)[d B]=10 \log P_{r}\left(d_{0}\right)-10 \log P_{r}(d)
$$

where $P_{r}(d)$ is the received power a distance $d$ from the transmitter. The reference distance $d_{0}$, which is taken here as $1 \mathrm{~m}$, is utilized to normalize the path loss to that which occurs at a distance $d_{0}$ from the transmitter so that only propagation effects are included in (1) [10].

Empirical or statistical approaches to predicting path loss then take the form

$$
\mathrm{PL}(d)[d B]=10 \log \left(\frac{d}{d_{0}}\right)^{n}+\sum_{p=1}^{P} \operatorname{WAF}(p)+\sum_{q=1}^{Q} \operatorname{FAF}(q)
$$

where $P$ and $Q$ are the number of walls and floors between the transmitter and the receiver respectively $[3,4]$. The empirical parameters $n, \operatorname{WAF}(p)$ and $\operatorname{FAF}(q)$ are termed path loss exponent, wall attenuation factor and floor attenuation factor respectively. The value of these parameters is determined by best fitting the model (2) to measurement data from the building of interest. The model (2) performs well in certain circumstances however its suffers from limitations. It does not include propagation effects such as distance dependence of path loss exponent, angle dependence of the WAF, reflection and diffraction. As a result prediction accuracy can be poor in certain parts of a building and especially at large distances from the transmitter. 


\section{The New Empirical Propagation Model}

To improve the accuracy of the empirical model (2) while retaining its simplicity we incorporate additional propagation effects that have been observed and suggested by UTD as described in the following sections.

\subsection{Distance Dependence of Path Loss Exponent}

It is observed that propagation loss as a function of distance has two distinct regions $[6,11]$. In the first region, which is within 5-20m of the transmitter propagation loss is similar to that occurring in free space. This is because at distances very near the antenna obstructions such as walls and floors do not interact significantly with the propagating waves. At distances further away in the next region, however, the propagation loss increases significantly as the electromagnetic waves become obstructed by the ceilings or walls of the rooms in the building. The distance at which this transition in propagation loss occurs is referred to here as the break point (this terminology is borrowed from microcells where a similar effect is observed [11]).

This break point phenomena is incorporated into (2) by modifying the equation to

$$
\begin{aligned}
\mathrm{PL}_{b p}(d)[d B]=10 \log \left(\frac{d}{d_{0}}\right)^{n_{1}} U\left(d_{b p}-d\right)+10\left[\log \left(\frac{d_{b p}}{d_{0}}\right)^{n_{1}}\right. & \left.+\log \left(\frac{d}{d_{b p}}\right)^{n_{2}}\right] U\left(d-d_{b p}\right) \\
& +\sum_{p=1}^{P} \operatorname{WAF}(p)+\sum_{q=1}^{Q} \operatorname{FAF}(q)
\end{aligned}
$$

where $d_{b p}$ is the distance of the break point from the transmitter, $n_{1}$ and $n_{2}$ are the path loss exponents either side of the break point and $U(\cdot)$ is the unit step function defined as

$$
U(d)= \begin{cases}0 & d<0 \\ 1 & d \geq 0\end{cases}
$$

The additional parameters $d_{b p}, n_{1}$ and $n_{2}$ we have introduced can be obtained from the use of Fresnel zones. By considering the size of the first Fresnel zone a distance $d$ from the transmitter and determining at what distance it will become obstructed the break point $d_{b p}$ can be calculated [7]. The diameter of the first Fresnel zone a distance $d$ from the transmitter is approximately given by $Z_{f} \approx \sqrt{\lambda d}$ as shown in [12]. Consequently in a corridor of width $1.5 \mathrm{~m}$ the first Fresnel zone will be obstructed at distances $d$, greater than about $7.5 \mathrm{~m}$ for frequencies around $900 \mathrm{MHz}$. In rooms where there is more clear space 
the first Fresnel zone will be obstructed by the ceiling or floor and this will occur at about $20 \mathrm{~m}$ from the transmitter. To keep our model simple we propose to take its value between these limits and use $10 \mathrm{~m}$ for the break point $d_{b p}$ in all situations.

The exponents $n_{1}$ and $n_{2}$ will also depend on the particular propagation environment. In general $n_{1}$ should be about the freespace value of 2.0 once antenna effects are removed [6]. The parameter $n_{2}$ should be greater than 2.0 since it represents propagation in which the first Fresnel zone is obstructed. We have found at $900 \mathrm{MHz}$ that $n_{2}$ is generally around 2.5 for propagation along a corridor [13].

\subsection{Angle Dependence of Attenuation Factors}

When electromagnetic radiation is incident on a wall or floor obliquely less power will be transmitted through the wall than would occur at normal incidence. To try and capture this effect in our new model we therefore propose to make the $\operatorname{WAF}(p)$ depend on the angle of incidence.

To quantify the dependence of the $\operatorname{WAF}(p)$ and $\operatorname{FAF}(q)$ on the angle of incidence we have investigated theoretically the loss you can expect for a variety of wall types. We calculated the loss by treating the

wall as a simple layered media, arriving at expressions for the transmission loss. Two examples of wall attenuation as a function of the angle of incidence are given in figure 1 . In general the exact expressions for the wall attenuation are algebraically non-trivial but the essence of them can be retained by using a simple approximation. At grazing incidence we assume that transmission is zero while at normal incidence we take transmission as the value of $\operatorname{WAF}(p)$ or $\operatorname{FAF}(q)$ that was originally obtained from propagation measurements as with (2). At angles between grazing and normal incidence we interpolate these values using a cosine function as $\operatorname{WAF}(p)[d B] / \cos \theta_{p}$ where the WAF $(p)[d B]$ is taken as the attenuation factor at normal incidence and $\theta_{p}$ is the angle of incidence at the $p^{t h}$ wall. The results of this approximation are also provided in figure 1 where it can be observed that it performs well especially when compared to what would be obtained if no variation of the $\operatorname{WAF}(p)$ with incident angle is allowed.

By incorporating this form for the attenuation factors into (3) we arrive at an improved model with very little increase in computational effort. The resulting model is given by

$$
\begin{aligned}
\mathrm{PL}_{\angle}(d)[d B]=10 \log \left(\frac{d}{d_{0}}\right)^{n_{1}} U\left(d_{b p}-d\right)+ & 10\left[\log \left(\frac{d_{b p}}{d_{0}}\right)^{n_{1}}+\log \left(\frac{d}{d_{b p}}\right)^{n_{2}}\right] U\left(d-d_{b p}\right) \\
& +\sum_{p=1}^{P} \mathrm{WAF}(p) / \cos \theta_{p}+\sum_{q=1}^{Q} \mathrm{FAF}(q) / \cos \theta_{q}
\end{aligned}
$$


where $\operatorname{WAF}(p)$ and $\operatorname{FAF}(q)$ are the values of the attenuation factors at normal incidence and the $\theta_{p}$ and $\theta_{q}$ are the angles respectively between the $p^{t h}$ wall and $q^{t h}$ floor and the straight line path joining the transmitter to the receiver. The subscript $\angle$ is used on PL to indicate that it is the path loss when the angle of incidence to the wall and the distance dependence of propagation is taken into account.

\subsection{Diffraction}

A significant limitation of the model (2) is that in practice propagation guided by corridors will sometimes provide an indirect path which may be significantly greater than the propagation loss predicted from the straight line path between the transmitter and receiver. Therefore indirect propagation paths need to be added to the model (2). The problem with trying to model indirect paths is that in general it is difficult to compute them without excessive computational load.

In our new model the method by which we try and approximate or compensate for the indirect path is to utilize one level of diffraction from corners (including door and window frames) in the building. To perform this we calculate the field due to the model (5) at each corner and determine the resulting diffracted field from them using a diffraction coefficient. This diffracted field will then act as a secondary source and propagates away from each corner by the same model as (5). Thus the total field at the receiver can be calculated as a summation of the field from the transmitter and all the corners.

To include these diffracted fields we invoke the diffraction coefficients for perfect electrical conductors under UTD and we write them as $D\left(d, \phi, d^{\prime}, \phi^{\prime}\right)$ where $(d, \phi)$ are the coordinates of the corner relative to the transmitter and $\left(d^{\prime}, \phi^{\prime}\right)$ are the coordinates of the receiver relative to the corner [14]. Our model can then be written as

$$
\operatorname{PL}_{D}(d, \phi)[d B]=-10 \log \left[\sum_{m=1}^{M}\left(\mathcal{P} \mathcal{L}_{L}\left(d_{m}\right) \mathcal{P} \mathcal{L}_{L}\left(d_{m}^{\prime}\right) \times\left|D\left(d_{m}, \phi_{m}, d_{m}^{\prime}, \phi_{m}^{\prime}\right)\right|^{2}\right)+\mathcal{P} \mathcal{L}_{L}(d)\right]
$$

where $M$ is the number of corners in the building database and the subscript $m$ refers to the $m t h$ corner,

$\mathcal{P} \mathcal{L}_{L}(\cdot)$ is the dimensionless quantity $10^{-P L} L^{(\cdot) / 10}$ and the final term in (6) takes account of the original straight line path from the transmitter to the receiver. It is also important to note that when we use (5) for the diffracted paths in (6) we set the path loss exponent $n_{1}$ to unity (we continue using $n_{1}=2.0$ for paths emanating directly from the transmitter). The reason is that the divergence factor (in terms of power) for diffracted fields is $\left[d / d^{\prime}\left(d^{\prime}+d\right)\right]$ and will effectively give a path loss exponent of $n_{1}=1.0$ when 
we are near a diffracting point $\left(d^{\prime}<d\right)[14]$. For $d^{\prime}$ greater than the breakpoint $d_{b p}$ we use the original $n_{2}$ since the Fresnel zone effects will be dominant.

\section{Propagation Prediction and Measurement Results}

Propagation predictions using the new model (6) and the conventional model (2) have been obtained in a variety of environments in Hong Kong and compared to actual measurements. In this paper we describe results from the Hong Kong University of Science and Technology.

The Hong Kong University of Science and Technology (HKUST) was completed in 1991 and consists of 7 floors and a total floor area of approximately $140,000 \mathrm{~m}^{2}$. The construction of the building is of concrete block and plaster board walls. In office and classroom areas the floor to floor height is $4 \mathrm{~m}$ with a suspended ceiling containing air-conditioning and service ducts $2.4 \mathrm{~m}$ above the floor. In laboratory areas the floors are reinforced with a floor to floor spacing of $5 \mathrm{~m}$ with a suspended ceiling $3 \mathrm{~m}$ above the floor. The general environment has furniture primarily constructed from wood or plastic in office, classroom and laboratory spaces.

To perform propagation prediction site specific information was obtained from the architect's AutoCAD building plans for HKUST by converting them into a format where the walls (and wall type), corners and floors had been identified. A crucial step in performing the propagation predictions is assigning values to the empirical parameters $n_{2}, d_{b p} \operatorname{WAF}(p)$ and $\operatorname{FAF}(q)\left(n_{1}=2.0\right.$ as described in section 3.1 and 3.3). In principle these parameters can be obtained from theoretical considerations or electromagnetic simulations. They can also be obtained by performing limited propagation experiments in the building and this is referred to as tuning.

For the tuning to be a practical procedure it is essential only limited propagation measurements are utilized and that these are from only a small area of the building. To tune $n_{2}$ and $d_{b p}$ we suggest acquiring propagation measurements along a corridor in which the transmitter is located at one end. The breakpoint and path loss exponents can then be found using a least squares best fit with the measurements (after any antenna effects associated with the pattern of the antennas are removed). To tune WAF $(p)$ we suggest placing the transmitter near to a region in the building which has several walls and measure the propagation loss between the walls along a line that is approximately perpendicular to the walls and 
passes through the transmitter location. Subsequently we estimate the WAF $(p)$ by best fitting to the model (3).

Prediction results for a $110 \times 25 \mathrm{~m}$ area of the EEE laboratories are provided in figure 2 at $900 \mathrm{MHz}$ where the transmitter is located in the lower left and marked by a cross. In figure 2 a the prediction results are for the new model (6) in which the parameters, $n_{2}=2.5, d_{b p}=10.0$ and WAF $=10 \mathrm{~dB}$ for concrete block walls (WAF $=5 \mathrm{~dB}$ for hollow plaster board walls), were obtained by tuning. In figure $2 \mathrm{~b}$ the results for the conventional model (2) are provided in which $n=2.4$ while the other parameters remain the same (these parameters were also found by tuning using the same set of limited measurements as the new model (6)). It can be observed that in the upper right region of the laboratory up to a 40dB difference in the predictions can be observed.

To compare our predictions to measurements, 287 samples of the actual field were acquired on an approximately uniform grid (with grid size of $5 \mathrm{~m}$ ) in the laboratory area at $900 \mathrm{MHz}$ with the transmitter in the same location as for the predictions. At each sample point the effects of fast fading were removed by using the mean value of at least 20 measurements in a $4 \mathrm{~m}^{2}$ area centered about the sample location. The propagation measurement equipment consisted of a fixed transmitter and a narrowband (30KHz) portable receiver with a notebook computer attached to allow data and position logging. The transmitting omni-directional (2.2dBi gain) antenna was attached to the suspended ceiling with the receiving omni-directional antenna (2.2dBi gain) at height $1.5 \mathrm{~m}$.

The comparisons with the predictions are presented in figure 3 as scatter plots. In figure 3 a the comparisons are for the new model (6) while in figure $3 \mathrm{~b}$ they are for the conventional model (2). Statistics of the comparisons are listed in table 1 . The standard deviation in error for the new model $(6)$ is $6.7 \mathrm{~dB}$ while that for the conventional model (2) is $20.8 \mathrm{~dB}$.

It can be observed that the conventional model (2) predictions overestimate the actual path loss by up to $40 \mathrm{~dB}$ as evidenced by the downward curving scatter plot in figure $3 \mathrm{~b}$. This overestimation corresponds to measurement samples from the upper right region of the floor plan where the actual path loss is largest. The reason the conventional model performs poorly in this region is because the direct path becomes blocked by the large number of walls between the transmitter and receiver making the conventional model predict a large propagation loss. The new model (6) however finds that the actual path loss is less because there is a diffracted path that exists from the door frames along the lower corridor through 
only two rooms to the upper corridor. The increase in accuracy demonstrated by the new model (6) is likely to reduce the number of basestations estimated by a planning tool [9], using the new model (6), as compared to estimations using the conventional model (2).

From measurements and predictions in other buildings around Hong Kong we have found similar trends. That is both models work well once tuned for regions nearby the transmitter (within 1-5 walls). However, in regions further away from the transmitter (greater than 5-10 walls) the direct path usually becomes blocked and only the new model provides useful results.

A potential limitation of our model is that it does not take account of reflected rays. For this reason we can expect the model to perform less well in highly reflective environments. However the actual reduction in accuracy may not be large as long as there are corners and edges in the building to cause diffraction. The reason is that around corners and edges the total field must remain continuous and consequently if the reflected fields are large the diffracted fields will be equally large along the shadow and reflection boundaries so that continuity is maintained. Consequently, diffraction will provide some indirect paths even in highly reflective environments if corners are present.

\section{Conclusions}

The contribution of the research presented here is that we have provided a model for propagation prediction that incorporates much of the propagation phenomena that is suggested by formal electromagnetic overheads without large computational overheads. We have also demonstrated that the model has improved accuracy compared to the conventional model in a variety of environments over large floor areas even in regions where indirect propagation effects are important.

Because of the low computation load of this approach it suggests that it would be ideally suited for incorporating into planning tools that automatically optimize the position of basestations within buildings $[9,15]$. 


\section{References}

[1] H. Hashemi, The indoor radio propagation channel, Proceedings IEEE, 81(7):943-968, July, 1993.

[2] D. Molkdar, Review on Radio Propagation into and within Buildings, IEE Proceedings Part H, 138(1):61-73, February 1991.

[3] S. Y. Seidel and T. S. Rappaport, 914 MHz Path loss Prediction models for Indoor Wireless Communications in Multifloored Buildings, IEEE Transactions on Antennas and Propagation, 40(2):207-217, February 1992.

[4] J.M. Keenan and A.J. Motley, Radio Coverage in buildings, British Telecom Technology Journal, 8(1):19-24, January 1990.

[5] J. W. McKown and R. L. Hamilton, Ray Tracing as a Design tool for Radio Networks, IEEE Network Magazine, pages 27-31, November 1991.

[6] W. Honcharenko, H. L. Bertoni and J. L. Dailing, Mechanisms governing Propagation between floors in buildings, IEEE Transactions on Antennas and Propagation, 41(6):787-790, June 1993.

[7] W. Honcharenko, H. L. Bertoni, J. L. Dailing, J. Qian and H. D. Yee, Mechanisms governing UHF Propagation on single floors in modern office buildings, IEEE Transactions on Vehicular Technology, 41(4):496-504, November 1992.

[8] T. S. Rappaport, S. Y. Seidel and K. R. Schaubach, Site-Specific Propagation prediction for PCS system design, In M. J. Feuerstein and T. S. Rappaport, editors, Wireless Personal Communications, pages 281-315, Kluwer Academic Publishers, 1993.

[9] R. D. Murch and K.W. Cheung, Optimizing Indoor Basestation Locations, In Abstracts of the XXVth General Assembly of the International Union of Radio Science, page 322, Lille, France, August 28 - September 5, 1997.

[10] D. M. J. Devasirvatham, A Comparison of Time Delay Spread and Signal Level Measurements within two dissimilar Office Buildings, IEEE Transactions on Antennas and Propagation, 35(3):319-324, March 1987.

[11] E. Green, Radio Link Design for microcellular systems, British Telecom Technology Journal , 8(1):8596, January 1990. 
[12] D. Parsons, editor, The mobile radio propagation channel, John Wiley and sons, New York, 1992.

[13] R. D. Murch, O.C.L Au, M.S.F. Yau, D.H.K. Tsang, T-M. Ko and R.W. Liu, Wireless Communications for the Hong Kong Environment: 1, Technical Report, Hutchison Telephone Limited, 23/F Citicorp Centre, 18 Whitfield Rd, North Point, Hong Kong, June 14, 1993.

[14] R. G. Kouyoumjian and P. H. Pathak, A Uniform Geometrical theory of diffraction for an edge in a perfectly conducting surface, Proceedings IEEE, 62(11):1148-1461, November 1974.

[15] H. D. Sherali, C. M. Pendyala and T. S. Rappaport, Optimal Location of Transmitters for MicroCellular Radio Communication System Design, IEEE Journal on Selected Areas in Communications, 14(4):662-673, May 1996. 


\begin{tabular}{|l|c|c|c|c|}
\hline Model & Mean error $(\mathrm{dB})$ & $\sigma$ error $(\mathrm{dB})$ & \% with error $< \pm 5 \mathrm{~dB}$ & \% with error $< \pm 10 \mathrm{~dB}$ \\
\hline New & -0.3 & 6.7 & 54.0 & 86.8 \\
\hline Conventional & 14.8 & 20.8 & 33.8 & 56.8 \\
\hline
\end{tabular}

Table 1: Statistics of comparisons in accuracy between the measurements (at 287 locations) and predictions by the new (6) and conventional (2) empirical models.

Figure 1: Wall attenuation as a function of the angle of incidence for two wall structures a) solid wall of thickness $12.5 \mathrm{~cm}$ and $\epsilon=3$, and b) hollow plaster wall with plaster board of thickness $1 \mathrm{~cm}$ and $\epsilon=3$ separated by an air gap of $10 \mathrm{~cm}$. The solid lines represent the attenuation calculated by treating a wall as a layered medium while the broken line is our approximation $\mathrm{WAF} / \cos \theta$.

Figure 2: Prediction results for a $110 \times 25 \mathrm{~m}$ section of HKUST in which the transmitter is located in the lower left corridor and is marked with a cross. The results are for a) new empirical model (6) and b) conventional model (2).

Figure 3: Scatter plot of predicted verses measured path loss. The scatter plots are for a) new empirical model (6) and b) conventional model (2). 


\section{Biography of K.W. Cheung}

Kwok-wai Cheung was born in Hong Kong and received the B. Eng. degree in Electrical and Electronic from King's College, University of London in 1990. In 1997, he received the Ph.D. degree in Electrical and Electronic Engineering from Hong Kong University of Science and Technology. Currently he is with Hong Kong Telecom working on propagation issues. His research interests include mobile radio propagation, detection/estimation and electromagnetic theory.

\section{Biography of J.H.M. Sau}

Jonathan H-M. Sau received a B.Sc.(Eng.) degree from the University of Hong Kong, Hong Kong in 1991, and an M.A.Sc. degree in Electrical Engineering from the University of Toronto, Canada in 1997. From 1993 to 1994, he was a Research Assistant in the Hong Kong University of Science and Technology. Currently he is with Motorola Information Products and Services Group, working on wireless data products. His research interests are CDMA system design, QoS in packet data networks and radio propagation prediction.

\section{Biography of R.D. Murch}

R. D. Murch (S'85-M'90) received his bachelors (with first class honors) and Ph.D degrees in Electrical and Electronic Engineering from the University of Canterbury, New Zealand in 1987 and 1990 respectively.

From 1990-92 he was a post doctorate fellow at the Department of Mathematics and Computer Science at Dundee University, Scotland. There he investigated new approaches to approximately calculating electromagnetic scattering from three-dimensional objects. He joined Hong Kong University of Science and Technology in 1992, where he is currently an Assistant Professor in the Department of Electrical and Electronic Engineering. Since 1994 he has also acted as a consultant for various industrial projects on wireless communications and has two U.S. patent applications in progress. His research interests include antennas and propagation, smart antenna systems and inverse scattering.

Dr. Murch is a Chartered Engineer, a member of IEE and an URSI correspondent. In 1993 and 1996 he won URSI Young Scientist and Engineering Teaching Excellence Appreciation Awards respectively. 
a)

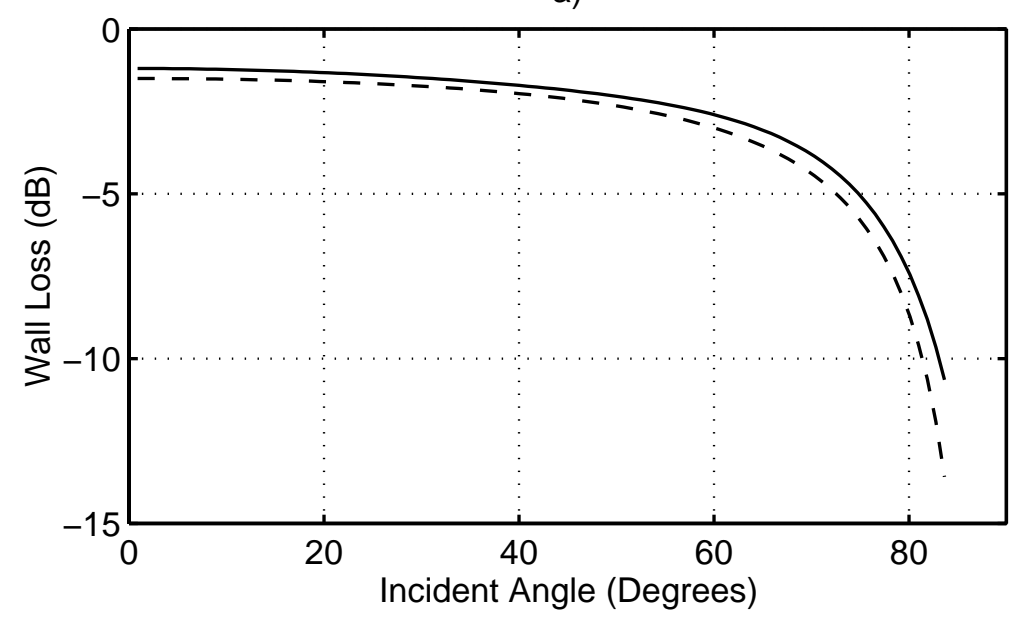

b)

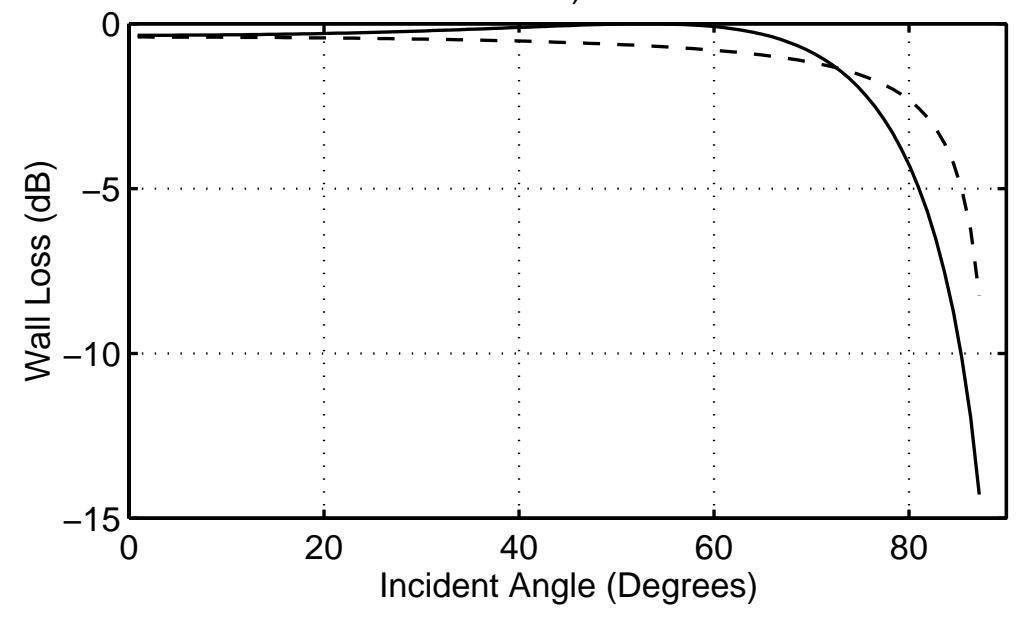

Figure 1: Wall attenuation as a function of the angle of incidence for two wall structures a) solid wall of thickness $12.5 \mathrm{~cm}$ and $\epsilon=3$, and b) hollow plaster wall with plaster board of thickness $1 \mathrm{~cm}$ and $\epsilon=3$ separated by an air gap of $10 \mathrm{~cm}$. The solid lines represent the attenuation calculated by treating a wall as a layered medium while the broken line is our approximation $\mathrm{WAF} / \cos \theta$. 
a)

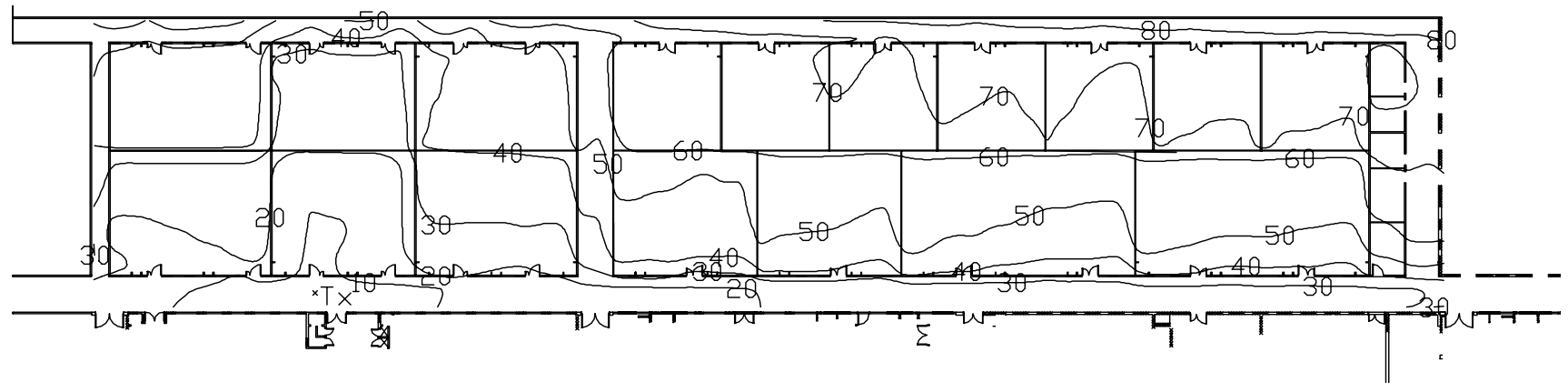

b)

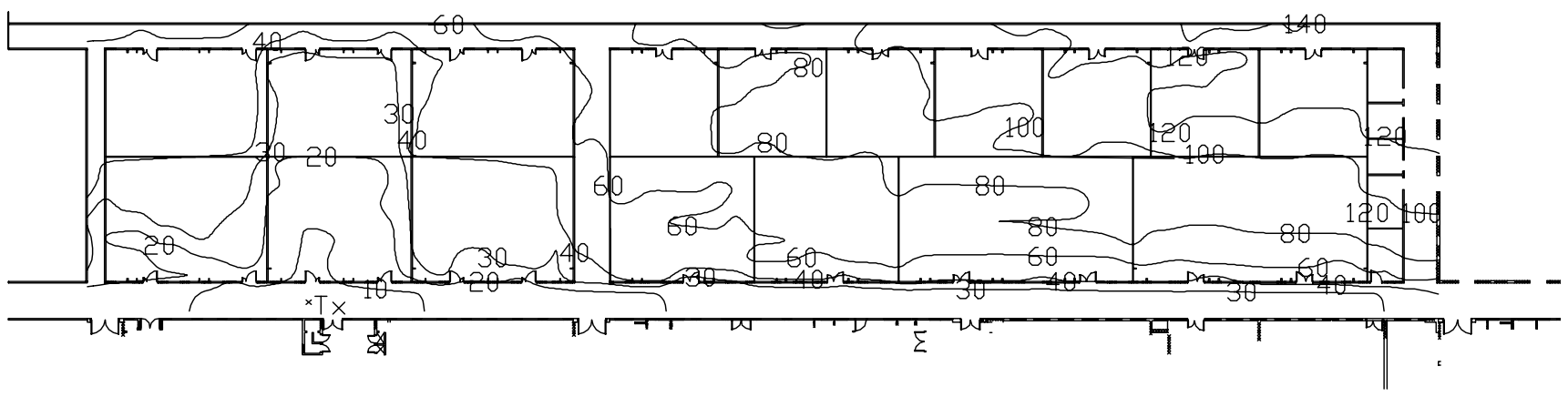

Figure 2: Prediction results for a $110 \times 25 \mathrm{~m}$ section of HKUST in which the transmitter is located in the lower left corridor and is marked with a cross. The results are for a) new empirical model (6) and b) conventional model (2). 
a)

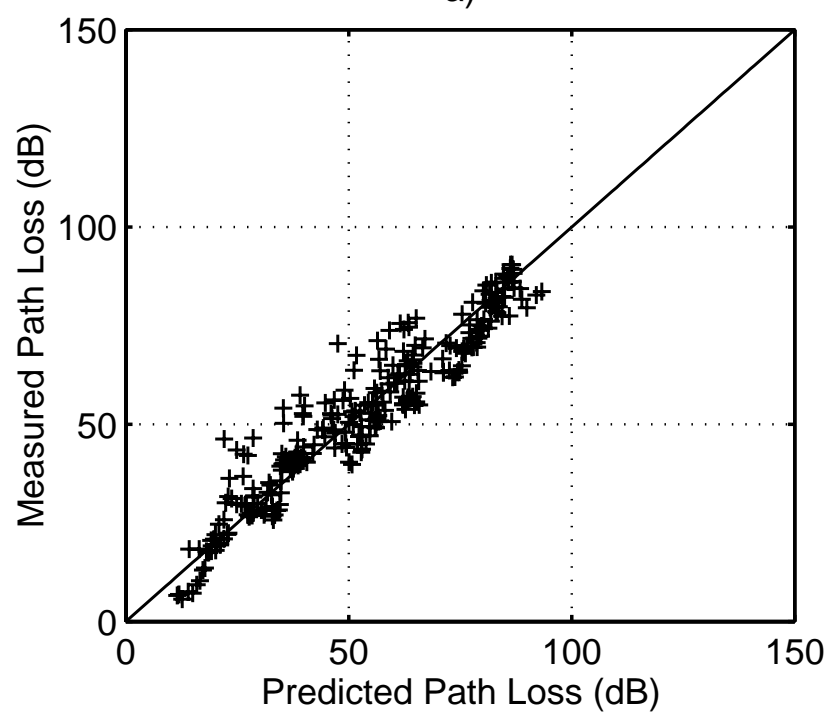

b)

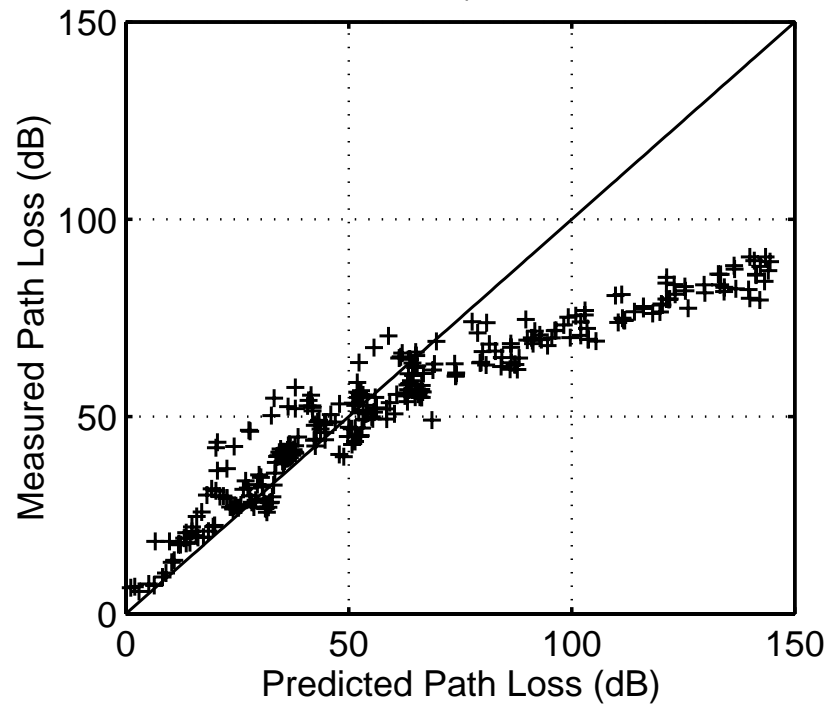

Figure 3: Scatter plot of predicted verses measured path loss. The scatter plots are for a) new empirical model (6) and b) conventional model (2). 